FEATURE ARTICLE

\title{
Nanotechnology: the science and engineering of tomorrow
}

\author{
S.R.P. Silva," N. Blanchard and S. Lyth \\ Advanced Technology Institute, University of Surrey, United Kingdom
}

Received : 13 February 2006; Accepted : February 2006

\section{What is Nanotechnology?}

Public awareness of nanotechnology has greatly increased in the early part of this new century, with numerous references in popular culture, most notably the "Grey Goo" of Michael Crichton's bestseller, Prey. There is great confusion and debate on how exactly to define nanotechnology, but for the purposes of this paper, we shall adopt the definition: science and engineering at the dimensions of one to one hundred nanometres $(\mathrm{nm})$, where a nanometre is one billionth of a metre.

To understand any development in technology, it is always useful to look to its history. We can trace the use of nanotechnology to the ancient Romans. When producing the $4^{\text {th }}$ Century Lycurgus Cup, the Romans used $70 \mathrm{~nm}$ silver colloids to produce colour and sparkle. Is it possible that the $5^{\text {th }}$ Century masterpieces at Sigiriya were painted using oil based paint, incorporated with metal nanoclusters?

Although the concept of the atom can be traced back to Greek antiquity, with Democritus of Adbera (400 BCE) asserting that all material things are composed of extremely small irreducible particles called atoms, it was not until the English chemist John Dalton (17661844) that a firm scientific theory of the atom began to develop. Many great scientists went further to contribute to our contemporary understanding of the world at the nanoscale, but this aspect will not be dealt with in this article, nevertheless we encourage the readers to read further on the subject.

It is the extraordinary Caltech physicist and Nobel laureate Richard Feynman (1918-1988) that many people cite as the inspiration for modern nanotechnology. In a lecture titled "There's Plenty of Room at the Bottom", presented to the American Physical Society in 1959, Feynman outlined the challenges and opportunities afforded by engineering starting from the atomic scale.

\section{The Nanotech Revolution: a panacea for humanities woes?}

The world we live in today has been altered dramatically by the seemingly ceaseless march of technology. Some would argue that this change has been for the worst, but most would agree that the quality of our lives has been vastly improved. The present state of affairs has been achieved through sudden bursts in technological output, often termed as "Revolutions". The first of these is the Industrial Revolution, which took place in Britain between 1780 and 1840 during which time the steam engine, textile industry were developed and much progress in mechanical engineering was achieved. This was closely followed by a second revolution in main land Europe (1840-1900) with the development of the railway infrastructure and the steel industry. Between 1900 and 1950 the hub of the third revolution was the USA, which resulted from the advent of the electric motor, the chemical industry, automobiles and various consumer goods. The fourth revolution (1950 to present), the Electronics Revolution, has been centred in the Pacific Basin (USA, Japan, Taiwan, South Korea), driven by Silicon Valley in California and the apparent insatiable appetites of consumers for ever more sophisticated electronic appliances.

This is where our story begins. It is the electronics industry that has, from a technological perspective pushed for ever increasing miniaturisation. The invention of the transistor by John Bardeen, Walter Brattain and William Shockley at Bell laboratories in 1947 , made it possible to start this revolution in miniaturisation. Jack Kilby and Robert Noyce took it a step further in the late 1950's when they both independently conceived the concept of the Integrated Circuit (IC), which allowed all the components of an electronic device to be fabricated on a single crystal of Silicon. This led Gordon Moore, the founder of INTEL, to predict in 1965 a doubling of the number of transistors 
per IC every 18 months. Moore's law relentlessly held for the last 40 years has been the driving force for change in IC design. Unfortunately, due to fundamental limits associated with quantum mechanical tunnelling in semiconducting and electronic materials it is envisaged that this miniaturisation of devices cannot continue beyond 2010. To overcome this barrier we must look to the potential offered by nanotechnology.

At the University of Surrey there are active research programmes in allotropes of carbon, specifically carbon nanotubes (CNT) and buckminsterfullerene $\left(\mathrm{C}_{6}\right)$. Research in this field began with the discovery of a stable molecule made up of 60 carbon atoms (Figure 1) by Kroto, Smalley and Curl in 1985. This finding led to them winning the 1996 Nobel Prize for Chemistry. This was closely followed by Iijimas identification of CNTs in 1991, creating a burgeoning field of research unparalleled in science. Why the big interest in $\mathrm{C}_{60}$ and CNTs?

The CNT, which is an elongation of the $\mathrm{C}_{60}$ molecule, exemplifies all that nanotechnology can offer (Figure 2). Identical in bonding to that found in soft metallic graphite, carbon nanotubes are single layers of graphitic carbon rolled up into a hollow tube, which is capped by half a football shaped $\mathrm{C}_{60}$ molecule. By virtue of its shape and bonding along its axis, this material is 20 times stronger than high tensile steel, half the weight of aluminium, has a current carrying capability a 1000 times greater than copper and can form either metallic or semiconducting derivatives based on how the tubes are rolled up. They also have a conduction mechanism that is not limited by the constraints of bulk materials and electrons travelling along the length of the tube do not

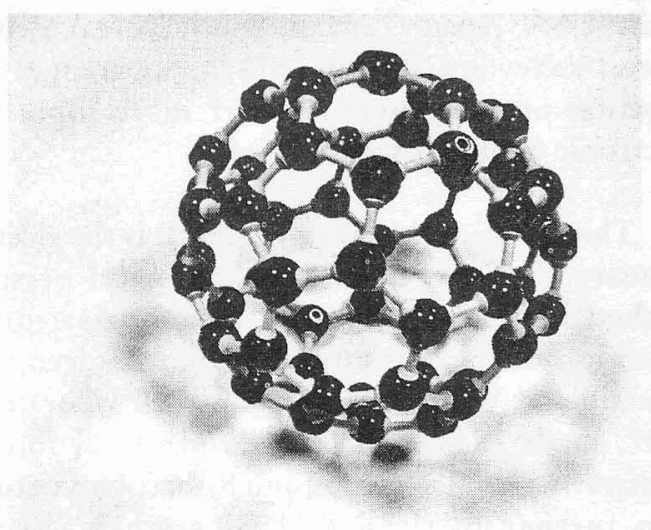

Figure 1: Ball and stick model of a C60 molecule experience electrical resistance in the same way as in bulk materials. This is known as ballistic transport and is possible due to the one dimensional quantum wire nature of the structure. In terms of heat dissipation, it has a thermal conduction twice that of diamond, once thought to be the best thermal conductor in the world.

The applications of CNTs in medical science, electronics, forensic science, and the environmental sciences could be far reaching and all pervasive. In the field of therapeutic medicine, buckyballs and CNTs offer ideal platforms for encapsulation of pharmaceuticals, enabling targeted drug delivery, thus reducing doses and removing their often harmful side-effects. In biology, the similar dimensions of DNA and nanostructures are proving to be an intriguing enabler that has given rise to nano-bio-technology. Lab-on-a-chip, DNA micro-arrays and novel diagnostics are all making the identification of genetic defects, pathogens and foreign matter cheaper, with increased flexibility and accessibility. In the future it is envisaged that we will be able to provide unobtrusive monitoring of patients in all environments with novel sensors for blood sugar, EEG, glucose, brain waves etc., on the end of say a 'stick-on-plaster', which may communicate with a processor in your watch.

Another aspect that captures the versatility of nanotechnology in its fullest sense is in the potential applications of Quantum Dots. Fundamentally, a quantum dot allows one to access the individual energy states associated with the band structure of materials. Therefore, instead of parabolic three dimensional energy states, now we have access to singular energy states such as those found in the hydrogenic model. This then allows us to very accurately utilise the energy transitions found

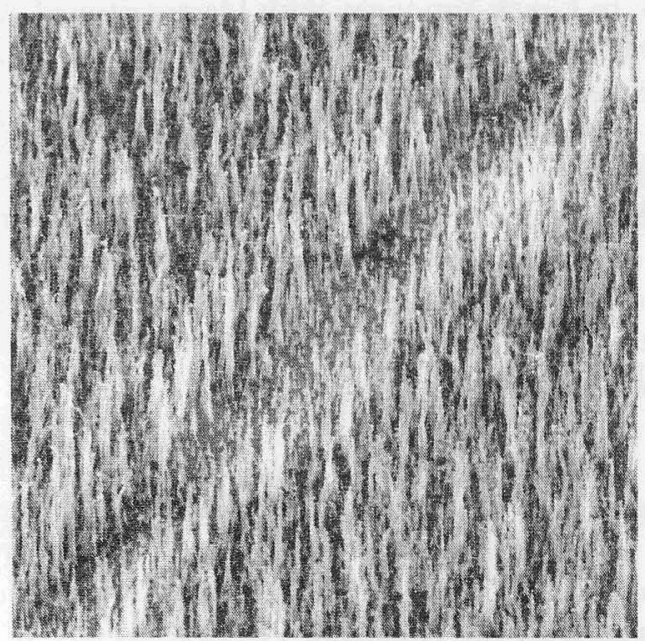

Figure 2: Thousands of carbon nanotubes can be grown over large areas. Each one of these tubes is about $500 \mathrm{~nm}$ long and about $80 \mathrm{~nm}$ thick. 
in quantum dots for energy capture and energy transfer situations. A typical example is in light harvesting from the sun in photovoltaics, where quantum dots are routinely used as a nano-composite material together with other organic compounds. We have found uses of quantum dots as light emitters and UV ray energy absorbers when combined with sun block creams. Another potential use is in accurate imaging of biological entities, where the quantum dot is tethered to a biomolecule. Laser devices based on quantum dots are also being pursued, provided suitably accurate manufacturing techniques for the growth of quantum dots is possible.

An extremely pertinent example where nanotechnology could have an enormous impact is energy. Only recently we have seen the tensions that can arise from energy demands, with the stand off between the Ukraine and the Russian gas company Gazprom. This could just be the beginning of such crises, particularly as demand for energy grows with fast developing economies such as China. There is also the environmental impact of our current reliance on fossil fuels. If there were sources of cheap renewable energy, it could develop the production base and economies of all countries, wipe out poverty and the east-west divide, and provide for medical care for the world population. We at Surrey are looking towards composites of CNTs and organic materials to produce high efficiency large area solar cells that could be produced on a roll-by-roll basis.

\section{The Nano-Nightmare!}

History has taught us that no technology comes without problems. The industrial revolution brought about excessive air pollution, culminating in the great smog of 1952 which killed an estimated 12,000 people in the city of London, UK. The methyl isocyanate gas leak in 1984 at the Union Carbide factory in Bhopal, India, killed 3,800 people and several thousand other individuals experienced permanent and partial disabilities. Perhaps the defining technology of the $20^{\text {th }}$ Century which still provokes great debate is that of nuclear power and weapons. There is no doubt that the world has been irrevocably changed by the bombs dropped on Nagasaki and Hiroshima in 1945, the "Cold War" (1945 - 1991), the Chernoblyl disaster in the Ukraine in 1986 and the continuing strife between the USA and Iran and North Korea. The one thing all these have in common is that the root of the dilemmas is not the technology but ignorance or irresponsible use.
What are the possible negative side-effects of nanotechnology? There has been a lot of news about self-replicating nano-bots that could render the whole surface of the earth to grey goo, but this is just sensationalism, and if such a scenario is possible it is a very long way off. A more imminent cause for concern is the toxicity of nano-particles, partly fuelled by health problems associated with asbestosis. Research has shown that nano-sized particles ingested in large quantities may accumulate in the nasal cavities, lungs and brains of rats and that 'buckyballs' induce brain damage in fish. However other studies show negligible effects. Further research on this aspect is needed and is being undertaken. As yet the yield in manufacture of nano-materials is low, thus making it expensive to produce the sizeable quantities that would be required to possibly enter the food chain.

\section{The future...}

Around the world many millions of dollars are being spent on research and technology to find competitive positions in this field. In 2003 alone, the US federal budget spent US $\$ 700 \mathrm{M}$, the UK government spent close to US\$170M, the Japanese and EU spent in excess of US $\$ 500 \mathrm{M}$. The state of California alone spent over US $\$ 100 \mathrm{M}$ for research. The US National Science Foundation predicts that the global market for nanotechbased products will exceed US $\$ 1$ trillion within 15 years.

Given the nature of nanotechnology, it is possible for developing nations to make a real impact. Thus not only will they benefit from the improved technology, but also build their economies by making an important contribution to the developments. In its 2005 report entitled "Innovation: applying knowledge in development", the UN Millennium Project task force on science, technology and innovation wrote that "nanotechnology is likely to be particularly important in the developing world, because it involves little labour, land or maintenance; it is bighly productive and inexpensive; and it requires only modest amounts of materials and energy".

What are the 'unknowns'? Is nanotechnology overhyped? Can it fulfil its promise without comprising society and security? Are its proponents realistic in their claims of what nanotechnology can achieve or is it a runaway technology destined to wreak havoc with human health and the natural environment? These are questions that can only be answered with time, but one thing is for sure, nanotechnology will achieve nothing without motivated scientists and engineers willing to take on the challenge. We hope this article will inspire you to take an interest in all areas of the science and maybe one day you could be the individual or more likely part of the team that answers these questions! 